\title{
Programme des Journées
}

(C) Société d'Anthropologie de Paris et Lavoisier SAS 2018

\section{MERCREDI 23 JANVIER : 9H30 - 12H15}

Le président, le secrétaire général, le comité scientifique et le comité d'organisation vous souhaitent la bienvenue aux $1844^{e}$ Journées de la Société d'Anthropologie de Paris.

09h30-10h20 Accueil café des participants

10h20-10h30 Ouverture par A. Balzeau, Président de la SAP et A. Delpuech, directeur du Musée de l’Homme

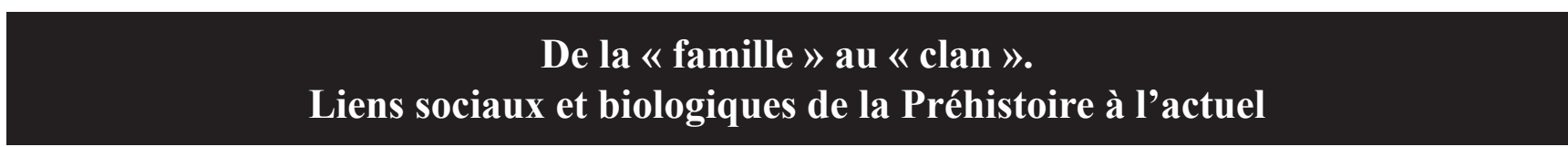

10h30-10h45 Qui rassemble-t-on dans les sépultures collectives et pourquoi ? - A. SCHMITT, S. DÉDERIX

10h45-11h00 Une approche archéogénétique de Fleury-sur-Orne, site à structures de type Passy (Normandie, Néolithique moyen) - M. Rivollat, P. Chambon, M.F. Deguilloux, E. Ghesquière, W. HaAk

11h00-11h15 La notion de famille chez les Gaulois : approche croisée de la génétique et de l'analyse spatiale C.E. Fischer, M. Le Roy, M.H. Pémonge, A. Lefort, C. Couture-Veschambre, S. Rottier, M.F. Deguilloux

11h15-11h30 Étude de l'organisation sociale de la production céramique des ateliers gallo-romain de Lezoux par l'analyse des traces digitales sur poterie - A. Lambert, A. Desmarais, C. Driard

11h30-11h45 Les pratiques funéraires du territoire de la civitas de Forum Lulii au cours du Haut-Empire : une approche archéothanatologique-A. Lattard, C. Gébara, M. Valente, P. Excoffon, J.C. Sourisseau, A. Schmit

11h45-12h00 Social structure of a I-II cc AD necropolis of "barbarians" of the Bosporan Kingdom near Bata (Novorossiysk, Russia) - A. Evteev, D. Barinov

12h00-12h15 Relation de proximité biologique au sein d'un échantillon médiéval provençal de statut social particulier (Saint Jean de Todon, Laudun l'Ardoise, Gard) - E. Verna, Y. Ardagna, C. Mopin, C. Rigeade, L. VIDAL 
MERCREDI 23 JANVIER : 14H30-17H45

De la « famille » au « clan ».

Liens sociaux et biologiques de la Préhistoire à l'actuel

14h30-14h45 Les apports de la paléogénomique à la caractérisation de l'impact de la colonisation russe sur les liens sociaux et biologiques en Yakutie - A. Seguin-Orlando, K. HanghøJ, C. Der Sarkissian, S. Duchesnes, P. Gérard, C. Thèves, A.N. Alexeev, E. Crubezy, MAfSO, the ANR LifeChange CONSORTIUM, L. OrLando

14h45-15h00 Impact de la patrilinéarité sur la diversité génétique des populations d'Asie Intérieure - E. HEYER, N. MARCHI

15h00-15h45 Communication invitée de R. CHaIx : Structures sociales et structures génétiques dans les populations humaines

(P) 15h45-16h15 : PAUSE

\section{Session posters}

16h15-17h45

SESSION POSTERS

(Voir la liste des posters en fin de programme) 
JEUDI 24 JANVIER : $10 \mathrm{H00}$ - $12 \mathrm{H} 45$

\section{Actualités de la recherche}

10h00-10h15 Middle Holocene ( 8300-3700 cal BP) hunter-gatherer dietary patterns in Cis-Baikal, Siberia A.W. Weber, R. Schulting, C.B. Ramsey, V.I. BaZaliskiI, O.I. Goriunova

10h15-10h30 Diet or Mobility? Multi-isotopic $\left({ }^{13} \mathrm{C},{ }^{15} \mathrm{~N}\right.$, and $\left.{ }^{87} \mathrm{Sr}\right)$ dietary modeling at Shamanka II, Cis-Baikal, Siberia - I. Scharlotta, T. Chaput, V.I. Bazalisski, G. Goude, S. Kusaka, A.W. Weber

10h30-10h45 Micro-sampling and early life dietary history of Early Bronze Age ( 4600-3700 cal BP) hunter-gatherers on Lake Baikal, Siberia, Russia - V. van der HaAs, V.I. BaZALIISKII, O. Goriunova, A.W. Weber

10h45-11h00 Changements alimentaires et culturels en Macédoine à l'âge du Bronze récent (1700/1500-1100 BCE, Grèce) - P. Tritsaroli, E. Herrscher, S. Koulidou, A. GuY

11h00-11h15 L'âge du Fer en Suisse occidentale : étude des conditions socio-économiques par l'analyse des isotopes stables du carbone et de l'azote $\left(\delta^{13} \mathrm{C}, \delta^{15} \mathrm{~N}\right)$ - J. Debard, J. Desideri, E. Herrscher, O. Dutour, M. BESSE

(P) $11 \mathrm{~h} 15-11 \mathrm{~h} 45:$ PAUSE

11h45 - 12h00 Paléo-oncologie au Pérou : deux cas de néoplasies de l'Horizon Moyen sur la côte centrale péruvienne C. Rojas-Sepúlveda, C. Bacigalupo

12h00 - 12h15 Les jeunes défunts du désert de Sechura (côte nord du Pérou) du Xe au XVe siècle (sociétés Lambayeque, Chimu-Inca) - L. Dausse, N. Goepfert, B. Gutiérrez, S. VÁsquez

12h15 - 12h30 Échanges biologiques polynésiens dans le sud-mélanésien ? Conjonction des données bio-anthropologiques et archéologiques de l'île de Futuna Ouest (Vanuatu) - W. Zinger, F. VAlentin, S. Bedford, J. Flexner, R. Shing, F. DÉtroit, D. Grimaud-Hervé

12h30 - 12h45 Étude paléogénétique des populations récentes et anciennes d'Asie centrale - P. GuARINo-VIGNon, C. Bon, E. Heyer, N. Marchi

12h45 - 13h15 VOTE POUR LE RENOUVELLEMENT DU CONSEIL D'ADMINISTRATION DE LA SAP

IO| 13h15 - 15h15 : DÉJEUNER 


\section{Assemblée générale de la SAP}

\section{5h15-16h15 ASSEMBLÉE GÉNÉRALE DE LA SAP}

L'AG est un temps fort de la vie de la SAP.

C'est une réunion consacrée aux bilans et à l'information.

Venez nombreux !

\section{Actualités de la recherche}

16h15-16h30 Les fossiles de Cro-Magnon (Dordogne, France) : 151 ans après leur découverte, et quelques nouveaux résultats - S. Villotte, A. Thibeault

16h30-16h45 Pratiques mortuaires et manipulations post-mortem au Gravettien : nouvelles données concernant les accumulations osseuses de la grotte de Cussac (Dordogne) - S. KaCki, E. Trinkaus, E.M.J. Schotsmans, I. Dori, P. Guyomarc'H, V.S. Sparacello, S. Villotte

16h45-17h00 Paléolithique final, premier et second Mésolithique : les partitions chronoculturelles de la fin du Pléistocène et du début de l'Holocène sont-elles pertinentes d'un point de vue de l'anthropologie biologique ? - M. SAMSEL

17h00-17h15 A new multidisciplinary analysis reveals diachronic information on Neolithic funerary behaviour and biocultural adaptations in Western Liguria (north-western Italy) - V. Sparacello, S. Rossi, C. Panelli, I. Dori, A. Varalli, G. Goude, J. Moggi-Cecchi, M. Conventi, D. Arobba, A. De Pascale, P. Garibaldi, G. Rossi, I. Molinari, M. Zavattaro, R. Maggi, E. Starnini, P. Biagi

17h15-17h30 Inhumés en silo de l'âge du Fer dans le Val d'Oise : tous malades ? - J.G. PARIAT, F. Boursier, E. Wermuth

17h30-17h45 Profil sanitaire d'une population provençale moderne et contemporaine : le cimetière Saint-Jacques à La Ciotat, Bouches-du-Rhône (1581-1831) - M. Perrin, Y. Ardagna, A. Richier, A. Schmitt 
VENDREDI 25 JANVIER : 10H00-13H00

\section{Correspondance génotype - phénotype : perspectives anthropologiques}

10h00-10h15 De la couleur de la robe des chevaux à l'évolution des populations humaines : illustration des études phénotypiques basées sur l'ADN ancien - M. Pruvost

10h15-10h30 Le flux de gène africain réduit l'anosmie à la beta-ionone chez une population malgache admixée H. Razafindrazaka, V. Pereda-Loth, C Ferdenzi, M. Heiske, C. Costedoat, M. Signoli, C. Radimilahy, T. Letellier, M. Bensafi, D. Pierron

10h30-10h45 Génétique du profil facial - B. Bonfante, A. Ruiz-Linares, C. Costedoat

10h45-11h00 Multivariate genotype-phenotype mapping (MGP): A new tool to explore the genetic architecture of human facial morphology - A. Le Maître, P. Mitteroecker

(P) 11h00-11h30 : PAUSE

11h30-11h45 Paléobiodiversité et évolution des hominines du Plio-Pléistocène à Sterkfontein, Afrique du Sud A. Beaudet

11h45-12h00 Variabilité intra-spécifique des processus de modelage osseux durant la croissance de l'os maxillaire chez Homo sapiens - A. Schuh, K. KupcziK, J.-J. Hublin, S. Freiduine

12h00-12h15 The association between craniometric and "neutral" biodistances in North Eurasia: the role of the scale of comparison - A. Evteev, A. Grosheva

12h15-13h00 Communication invitée de J.T. Richtsmeier : From genotype to phenotype and back again 
VENDREDI 25 JANVIER : 15H00- $16 \mathrm{H30}$

\section{Actualités de la recherche}

15h00-15h15 Relation entre capacités cognitives et écologie alimentaire chez l'Homme et quatre espèces de primates non-humains : nouvelles perspectives - M. Louail, E. Gilissen, S. Prat, C. Garcia, S. Bouret

15h15-15h30 Stratégie de vie chez les pygmées Baka : mortalité, fertilité et l'impact de l'alcool - F. RamiRez RozzI

15h30-15h45 Différences cinématiques de la cage thoracique chez les hommes et les femmes adultes en 3D D. García-Martínez, M. Bastir, N. Torres-Tamayo, I. Torres-Sánchez, F. García-Río, Y. Heuzé

15h45-16h00 Modèles de perturbations de croissance à partir de l'analyse de l'hypoplasie linéaire de l'émail (via macrophotographie) dans un échantillon néolithique de Ligurie (nord-ouest de l'Italie) - E. Orellana González, V.S. Sparacello, E. Bocaege, A. Varalli, J. Moggi-Cecchi, I. Dori

16h00-16h15 Visualisation non-destructive des anneaux du cément par microtomographie synchrotron : implications pour l'estimation de l'âge à la mort des adultes - A. Le Cabec, N.K. Tang, V. Ruano Rubio, S. Hilluson

16h15-16h30

Remise du Prix de la SAP et du Prix du poster. Clôture

Merci beaucoup pour votre participation et rendez-vous l'année prochaine pour les

$1845^{e s}$ Journées de la Société d'anthropologie de Paris, qui auront lieu

à Aix-en-Provence du 29 au 31 janvier 2020 


\section{Posters}

Session 2

2-1

Session 3

3-1

3-2

3-3

3-4

3-5

3-6

3-10

3-11

3-12

3-13

De la « famille » au « clan ». Liens sociaux et biologiques de la Préhistoire à l'actuel

La contribution de la paléo-alimentation à la compréhension des relations socio-culturelles préhistoriques : une étude de cas en l'Italie centrale à l'Âge du Cuivre - S. Bernardini, C. Conati Barbaro, J. Moggi-Cecchi, M.A. TAfuri

\section{Actualités de la recherche}

Un probable cas tardo-antique de Maladie de Paget dans le Haut-Var (Nécropole de la Rue Louis Cauvin, Garéoult Var) - Y. Ardagna, E. Spérandio, E. Verna, A. Acovitsioti-Hameau, M. Panuel, P. HaMeau

Les sépultures de Ngongo Mbata (RDC, XVII-XIXe siècles) : recrutement et état sanitaire - S. AsTI, C. Polet, A. Kharobi, P. Courtaud

La pneumatisation de l'os temporal : le cas d'Australopithecus sediba et implications pour la définition du genre Homo - A. Balzeau

Étude du microbiote ancien à partir de l'ADN ancien appliqué à la civilisation de l'Oxus à l'Âge de Bronze - M. Borry, C. Bon, M. Mashkour, J. Bendezu-Sarmiento

Variabilité des conformations des mandibules humaines du Pléistocène moyen en Europe : le cas de la mandibule de Montmaurin la Niche (Haute-Garonne, France) - C. Champalle, A. Vialet

Can Probabilistic Sex Diagnosis be used on Pre-Columbian mummies? - T. Chapman, C. Tilleux, C. Polet, J.P. Hastir, E. Coche, S. Lemaitre

Les crémations protohistoriques sur le site du Vigneau (Pussigny, Indre-et-Loire) - E. ChоL, A. Hauzer, A. Coutelas

Trépanation versus pseudo-trépanation : réviser les critères diagnostiques - C. JENGER, C. Partiot, A. Thomas

Dimensions dentaires et taille corporelle, des rapports qui dépendent de la population - F. RAMIREZ Rozzi, A. Romero

Déterminer le sexe des squelettes grâce à la génétique : comment et à quel prix ? - J. SAuvage, C. Bon, A. Тномаs

Apport de la micro-tomodensitométrie et de l'imagerie 3D à l'étude de trépanations néolithiques et médiévales en Belgique - J. Simons, C. Polet, O. Dutour, H. Coqueugniot

Modélisation de la langue dans la cavité buccale. Application aux Néandertaliens - A.Vialet, A. BiJar, Y. Payan, P. Perrier, D. Grimaud-Hervé, P. Frey, L. Norgeot

Le site archéologique Ngetepeu (Rép. centrafricaine) : vestiges osseux - H. Zana, L. Sumani, J. Sadoine, F. Ramirez Rozzi 